\title{
Brolucizimab-leading an era of structural revolution for long-term VEGF suppression
}

\author{
Ashish Sharma $^{1} \cdot$ Nilesh Kumar $^{1}{ }^{1} \cdot$ Baruch D. Kuppermann ${ }^{2} \cdot$ Francesco Bandello $^{3}$
}

Received: 9 July 2019 / Accepted: 26 July 2019 / Published online: 19 September 2019

(C) The Royal College of Ophthalmologists 2019

Biologics since their inception have been the breakthrough treatment modality for many retinal pathologies such as neovascular age-related macular degeneration (nAMD), diabetic macular edema, retinal vein occlusion etc. [1]. It has been long hypothesized that the modular nature of these molecules can be exploited to deliver customized therapeutics with specific pharmacological properties like valency, affinity, and tissue penetration intended for the specific pathology [2].

These biologics have varied molecular structure targeting VEGF molecule because VEGF-VEGF receptor (VEGFR) pathway is one of the main pathological pathways recruited in the majority of retinal vascular pathologies. VEGF-A, member of the VEGF proteins family, plays the central role in the angiogenic pathway. VEGF-A binds to VEGFR1 and VEGFR2 and causes stimulation of endothelial cells, macrophage migration, increased vascular permeability, and causes the breakdown of blood-retinal barrier. The VEGF-A is encoded by a single gene and thus by variable gene-splicing during expression, several isoforms of VEGFA exist, out of which VEGF-165 is the most abundant and potent. Other pathways include Angiopoietin-Tie receptor axis, Ephrin-Eph pathway, Delta-Notch pathway, as well as employs insulin-like growth factor-1 and interleukin-8 [1].

Ophthalmology holds a unique place in the development and adoption of these biologics. The molecular structures of the drugs aimed for intravitreal use range from RNA aptamers (pegaptanib) to full-sized monoclonal antibodies

Ashish Sharma

drashish79@hotmail.com

1 Department of Vitreoretina, Lotus Eye Hospital and Institute, Coimbatore, India

2 Gavin Herbert Eye Institute, University of California, Irvine, CA, USA

3 Department of Ophthalmology, University Vita-Salute Scientific Institute San Raffaele, Milano, Italy
(mAb: bevacizumab) to Fab fragments (ranibizumab) to antibody conjugate (aflibercept). In addition, single-chain variable fragment (scFv: brolucizumab), bispecific monoclonal antibody (faricimab), and DARPin (abicipar pegol) are in an advanced phase of clinical trials and are showing promising results. All of these biologics are intended to be used intravitreally for similar indications like nAMD, and thus provide a unique opportunity for a head-to-head analysis of drugs with different structures and their efficacy. With the advent of optical coherence tomography of the retina, the in vivo pathological status of the disease and the effect of these drugs on the pathology can be studied, recorded, and compared across the timeline. The changes in levels of intraretinal fluid, subretinal fluid are the direct indicator for the tissue penetration capabilities of these molecules [3]. Thus, ophthalmologists are poised to lead the innovation by providing real-world feedback to the antibody drug designers.

The varying drug structure has many pharmacokinetic implications. The antibody fragments such as ranibizumab, brolucizumab have a higher molar concentration of the drug, better tissue penetrance, and less immunogenicity risk [4]. The antibody fragments also have the ability to be linked to other fragments leading to bispecific, multispecific, multimeric, or multifunctional molecules [2]. Pegaptanib is a 28-base RNA aptamer, which selectively binds to VEGF-A 165 and prevents its binding to VEGFR1 [5]. Bevacizumab is used as an off-label drug for retinal neovascularization, is a full-sized humanized anti-VEGF monoclonal antibody, while ranibizumab is a humanized Fab fragment that neutralizes soluble splice variants and proteolytic fragments of VEGF-A. Aflibercept (VEGF trap) is a chimeric molecule carrying soluble receptors VEGFR1 and VEGFR2 linked to an Fc fragment of human IgG1, which essentially binds to VEGF and prevents its action [5]. Brolucizumab is a $28 \mathrm{kDa}$ humanized $\mathrm{scFv}$ molecule that binds to all the isoforms of VEGF-A and renders them ineffective [3]. Faricimab is a bispecific molecule bound to an optimized Fc fragment that has anti-VEGF-A and 


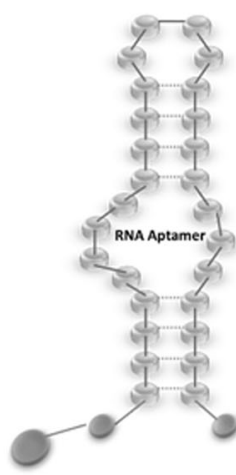

Pegaptanib

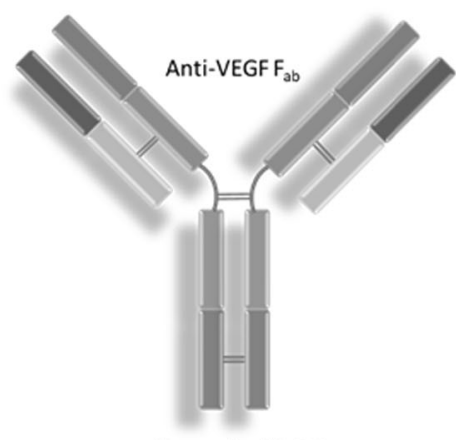

Humanized IgG $F_{c}$

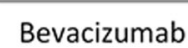

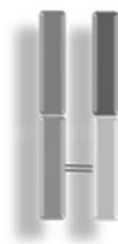

Anti-VEGF $F_{a b}$

Ranibizumab

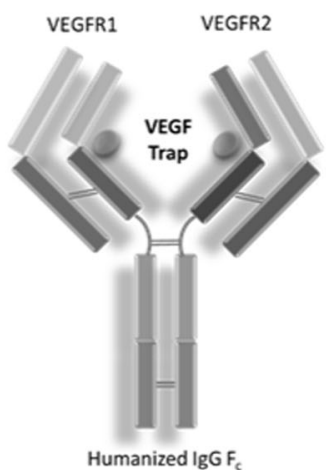

Humanized IgG $F_{c}$

Aflibercept

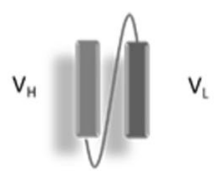

scFv

Brolucizumab
Anti-VEGF $F_{a b}$

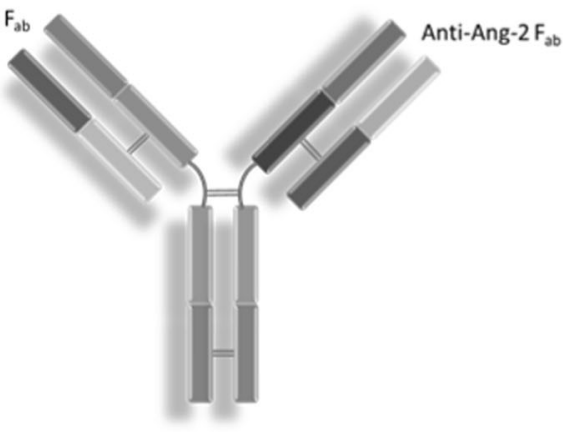

Humanized IgG $F_{c}$

Faricimab

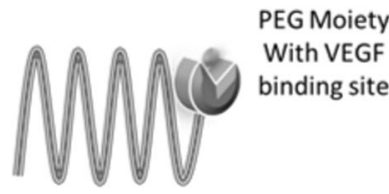

Ankyrin Repeats

Abicipar Pegol (DARPin)

Fig. 1 Variation in molecular structure of originator biologics used in ophthalmology

anti-Ang2 action and thus targets a major and a minor pathway of retinal neovascularization [6]. The abicipar pegol, in contrast, is a novel class of engineered ankyrin repeat domain that bind to VEGF-A with high affinity and specificity [7]. Recent trials have shown faricimab (BOULEVARD) to give better visual outcomes than ranibizumab, while brolucizumab (HAWK, HARRIER) provided a significantly longer duration of action than aflibercept $[3,6]$. Abicipar in initial trials was found to have 100-fold greater affinity than ranibizumab (Fig. 1) [8].

Absence of the Fc region in the smaller molecules is thought to be advantageous. Fc region binds to various receptors and leads to activation of bystander immune cell activation and antibody effector functions such as antibodydependent cellular cytotoxicity, antibody-dependent cellular phagocytosis, or complement-dependent cytotoxicity. The absence of Fc region thus leads to fewer side effects [4]. Fc region though binds to FcR receptors and so takes a long time for systemic clearance, while smaller molecules have rapid renal clearance. A recent head-to-head analysis by Joo et al. of a novel VEGF trap molecule with and without its Fc region showed that absence of $\mathrm{Fc}$ region leads to a longer intravitreal life, though the absence of Fc region makes the fragments more susceptible for aggregation and are thermolable [9]. The fusion of $\mathrm{scFv}$ with albumin or polyethylene glycol leads to better stability but also reduces the economic advantage of the molecule [10, 11]. Abicipar pegol is a $14 \mathrm{kDa}$ repeat ankyrin molecule that is attached with a $20 \mathrm{kDa}$ polyethylene glycol moiety, which binds to soluble VEGF molecules [7].

The smaller antibody fragments do not only hold pharmacokinetic advantage but also have a relatively easier manufacturing process. The full $\mathrm{mAb}$ requires a mammalian cell line for production, while $\mathrm{scFv}$ can be produced on the genetically engineered bacterial cell line $[12,13]$. The $\mathrm{V}_{\mathrm{H}}$ and $\mathrm{V}_{\mathrm{L}}$ chains of brolucizumab are genetically linked, and this unique structure can be expressed from a single genetic transcript, without balancing the production of different components as required for a full mAb. This gives faster turnover, higher yield, easier handling, and has relatively less manufacturing cost. Reduced cost of manufacturing coupled with less frequent injection requirements positions the smaller molecules like brolucizumab as a better option to combat retinal neovascularization. 


\section{Compliance with ethical standards}

Conflict of interest The authors declare that they have no conflict of interest.

Publisher's note Springer Nature remains neutral with regard to jurisdictional claims in published maps and institutional affiliations.

\section{References}

1. Keane PA, Sadda SR. Development of anti-VEGF therapies for intraocular use: a guide for clinicians. J Ophthalmol. 2012;2012: $1-13$.

2. Bates A, Power CA. Goliath: the structure, function, and clinical prospects of antibody fragments. Antibodies. 2019;8:28.

3. Dugel PU, Koh A, Ogura Y, Jaffe GJ, Schmidt-Erfurth U, Brown DM, et al. HAWK and HARRIER: phase 3, multicenter, randomized, double-masked trials of brolucizumab for neovascular agerelated macular degeneration. Ophthalmology. 2019; pii: S01616420(18)33018-5.

4. Kholodenko RV, Kalinovsky DV, Doronin II, Ponomarev ED, Kholodenko IV. Antibody fragments as potential biopharmaceuticals for cancer therapy: success and limitations. Curr Med Chem. 2019;26:396-426.

5. Amadio M, Govoni S, Pascale A. Targeting VEGF in eye neovascularization: what's new? Pharmacol Res. 2016;103: 253-69.
6. Sahni J, Patel SS, Dugel PU, Khanani AM, Jhaveri CD, Wykoff $\mathrm{CC}$, et al. Simultaneous inhibition of angiopoietin-2 and vascular endothelial growth factor-A with faricimab in diabetic macular edema. Ophthalmology. 2019;126:1155-70.

7. Rodrigues GA, Mason M, Christie L-A, Hansen C, Hernandez LM, Burke J, et al. Functional characterization of abicipar-pegol, an anti-VEGF DARPin therapeutic that potently inhibits angiogenesis and vascular permeability. Investig Ophthalmol Vis Sci. 2018;59:5836.

8. Callanan D, Kunimoto D, Maturi RK, Patel SS, Staurenghi G, Wolf $\mathrm{S}$, et al. Double-masked, randomized, phase 2 evaluation of abicipar pegol (an anti-VEGF DARPin therapeutic) in neovascular age-related macular degeneration. J Ocul Pharmacol Therapeutics. 2018;34:700-9.

9. Joo K, Park SJ, Choi Y, Lee JE, Na YM, Hong HK, et al. Role of the $\mathrm{Fc}$ region in the vitreous half-life of anti-VEGF drugs. Investig Ophthalmol Vis Sci. 2017;58:4261.

10. Jain A, Jain SK. PEGylation: an approach for drug delivery. A review. Crit Rev Ther Drug Carr Syst. 2008;25:403-47.

11. Müller D, Karle A, Meissburger B, Höfig I, Stork R, Kontermann RE. Improved pharmacokinetics of recombinant bispecific antibody molecules by fusion to human serum albumin. J Biol Chem. 2007;282:12650-60.

12. Montoliu-Gaya L, Esquerda-Canals G, Bronsoms S, Villegas S. Production of an anti-A $\beta$ antibody fragment in pichia pastoris and in vitro and in vivo validation of its therapeutic effect. PLOS ONE. 2017; 12:e0181480.

13. Spadiut O, Capone S, Krainer F, Glieder A, Herwig C. Microbials for the production of monoclonal antibodies and antibody fragments. Trends Biotechnol. 2014;32:54-60. 